ver. 2.1

\title{
Suzaku Observation of Strong Fluorescent Iron Line Emission from the Young Stellar Object V1647 Ori during Its New X-ray Outburst
}

\author{
Kenji Hamaguchi ${ }^{1,2}$, Nicolas Grosso ${ }^{3,4}$, Joel H. Kastner ${ }^{5}$, David A. Weintraub ${ }^{6}$, Michael \\ Richmond $^{5}$
}

\begin{abstract}
The Suzaku X-ray satellite observed the young stellar object V1647 Ori on 2008 October 8 during the new mass accretion outburst reported in August 2008. During the $87 \mathrm{ksec}$ observation with a net exposure of $40 \mathrm{ks}$, V1647 Ori showed a high level of X-ray emission with a gradual decrease in flux by a factor of 5 and then displayed an abrupt flux increase by an order of magnitude. Such enhanced X-ray variability was also seen in XMM-Newton observations in 2004 and 2005 during the 2003-2005 outburst, but has rarely been observed for other young stellar objects. The spectrum clearly displays emission from Helium-like iron, which is a signature of hot plasma $(k T \sim 5 \mathrm{keV})$. It also shows a fluorescent iron $\mathrm{K} \alpha$ line with a remarkably large equivalent width of $\sim 600 \mathrm{eV}$. Such a large equivalent width suggests that a part of the incident X-ray emission that irradiates the circumstellar material and/or the stellar surface is hidden from our line of sight. XMM-Newton spectra during the 2003-2005 outburst did not show a strong fluorescent iron $\mathrm{K} \alpha$ line, so that the structure of the circumstellar gas very close to the stellar core that absorbs and re-emits X-ray emission from the central
\end{abstract}

\footnotetext{
${ }^{1}$ CRESST and X-ray Astrophysics Laboratory NASA/GSFC, Greenbelt, MD 20771; Kenji.Hamaguchi@nasa.gov.

${ }^{2}$ Department of Physics, University of Maryland, Baltimore County, 1000 Hilltop Circle, Baltimore, MD 21250 .

${ }^{3}$ Université de Strasbourg, Observatoire Astronomique de Strasbourg, 11 rue de l'université, 67000 Strasbourg, France

${ }^{4}$ CNRS, UMR 7550, 11 rue de l'université, 67000 Strasbourg, France

${ }^{5}$ Rochester Institute of Technology, 54 Lomb Memorial Drive, Rochester, NY 14623.

${ }^{6}$ Vanderbilt University, Nashville, TN 37235.
} 
object may have changed in between 2005 and 2008. This phenomenon may be related to changes in the infrared morphology of McNeil's nebula between 2004 and 2008 .

Subject headings: stars: formation — stars: individual (V1647 Ori) — stars: pre-main sequence - X-rays: stars

\section{Introduction}

Certain young stars dramatically increase their mass accretion rates by orders of magnitude (from $10^{-7} M_{\odot} \mathrm{yr}^{-1}$ to $10^{-4} M_{\odot} \mathrm{yr}^{-1}$ ). These events are possibly triggered by thermal disk instabilities and are traced by dramatic increases in optical/IR luminosities; Such eruptive pre-main sequence (PMS) stars are crudely classified as either FU Ori (FUor) or EX Lupi (EXor) types; the former are characterized by outbursts lasting decades, whereas the latter generally display shorter outbursts, of duration a few months or years (see reviews in Hartmann \& Kenyon 1996). Although PMS stars are known to be luminous X-ray sources, FUor or EXor events have been reported only rarely, such that the level of PMS X-ray activity during an outburst is poorly established.

The young stellar object (YSO) V1647 Ori, which is deeply embedded in the L1630 dark cloud ( $d \sim 400$ pc, Anthony-Twarog 1982), underwent a strong optical/NIR outburst in 2003 December. This eruption afforded the first opportunity to record the sustained X-ray outburst of a rapidly accreting YSO (Kastner et al. 2004; Grosso et al. 2005; Grosso 2006; Kastner et al. 2006). Multiple Chandra and XMM-Newton observations through this outburst demonstrated that the average X-ray flux varied in the same way and on approximately the same timescale as the optical and IR brightnesses, although the X-ray flux also varied strongly (by a factor of up to 20) on timescales of less than a day. During outburst, the V1647 Ori X-ray spectrum had the characteristics of deeply embedded hot plasma $\left(N_{\mathrm{H}}\right.$ $\left.\sim 4.1 \times 10^{22} \mathrm{~cm}^{-2}, k T \sim 4.2 \mathrm{keV}\right)$. This result appears to be explained best as star-disk magnetic reconnection activity generated in association with the episode of very rapid mass infall (Kastner et al. 2004, 2006).

Following the V1647 Ori event, the EXor candidate V1118 Ori recently displayed a mass accretion outburst; followup X-ray observations of this object detected a moderate enhancement in the X-ray flux that was correlated with the optical/IR flux (Audard et al. 2005; ;udard et al. 2009). Skinner et al. (2006, 2009) reported that two FUor systems with ongoing historical outbursts, FU Ori and V1735 Cyg, showed very hard spectra of $k T \gtrsim 5 \mathrm{keV}$ plasmas or equivalent despite relatively steady X-ray fluxes. Systematic surveys of low-mass 
PMS (T Tauri) stars in the Orion nebula and the Taurus dark cloud (e.g., Preibisch et al. 2005; ;elleschi et al. 2007) indicate that accreting T Tauri stars are less X-ray active (by a factor of $\sim 2-3$ on average) than non-accreting $\mathrm{T}$ Tauri stars, suggesting that mass accretion activity may in fact suppress X-ray activity. Hence, the relationship between PMS mass accretion and high energy radiation remains unclear.

V1647 Ori began a new optical/NIR outburst at a certain time between 2008 Jan. 2 and Aug. 26 (Itagaki et al. 2008; Aspin 2008; Aspin et al. 2009). Our team later triggered an anticipated Target of Opportunity (ToO) observation of V1647 Ori with Chandra on September 18 for $20 \mathrm{ksec}$, in which the X-ray count rate was double the level observed by Chandra on 2004 March 7 (Kastner et al. 2004) during the previous outburst in 2003-2005 (Weintraub et al. in preparation). This new X-ray eruption offers us the first (and possibly unique) opportunity to measure X-ray emission during two outbursts from the same YSO. We therefore proposed a ToO observation with the Suzaku X-ray observatory (Mitsuda et al. 2007), so as to obtain detailed X-ray spectral diagnostics and a $\sim$ day timescale light curve of V1647 Ori during its latest accretion outburst. With this information, we can derive the hottest plasma temperature and the intrinsic activity variation of V1647 Ori, unaffected by circumstellar absorption; we can also constrain models of the plasma geometry and of the response of neutral disk material to X-ray irradiation. This paper focuses on the results of the Suzaku observation.

\section{Observation}

Suzaku observed V1647 Ori for 87 ksec on 2008 October 8, when the YSO's optical/NIR fluxes leveled off at a brightness similar to that observed in 2004 Feb.-Mar. (Ojha et al. 2008; Aspin \& Reipurth 2009). At the time of this observation, the observatory operated with three sets of telescope and detector systems - the thin-foil X-Ray Telescope (XRT) module (Serlemitsos et al. 2007) with the X-ray CCD detector (XIS: X-ray Imaging Spectrometer, Kovama et al. 2007) on its focal plane, and the Hard X-ray Detector (HXD: Takahashi et al. 2007; Kokubun et al. 2007) with narrow collimator arrays. The XRT+XIS system focuses X-rays with a half-power diameter (HPD) of $\sim 2^{\prime}$ and covers the X-ray energy range up to $\sim 10 \mathrm{keV}$. The XIS1 camera employs back-illuminated (BI) CCDs for the soft band sensitivity down to $\sim 0.3 \mathrm{keV}$, while the other XIS cameras (XIS0, XIS3) use front-side illuminated (FI) CCDs with sensitivity down to $\sim 0.4 \mathrm{keV}$. The HXD detects hard X-rays above $15 \mathrm{keV}$.

We started analysis from the cleaned event data in the distribution package, preprocessed with the version 2.2.11.22. The cleaned data excluded events recorded during 
high background periods, mainly when the satellite passed the South Atlantic Anomaly (SAA) 9 times during this observation, and earth occultations, each of which happened for $\sim 2.1 \mathrm{ksec}$ in every Suzaku orbit $(\sim 5.4 \mathrm{ksec})$. The data did not suffer telemetry saturation. We thus selected events in good time intervals of all the XIS0, 1 and 3 sensors. After this selection, the net exposure was 40,446 sec, i.e., $\sim 46 \%$ of the observing time. For the XIS arf generation, we used the CALDB version released on 2009-02-03, to better reproduce the recent growth of contamination on the XISs, in particular XIS0. The FI (XIS 0+3) and BI sensors collected 1216 and 624 counts between 1-8 keV, which include particle background and contamination from nearby sources by $\sim 26 \%$ and $\sim 39 \%$, respectively.

We generated time-averaged HXD spectra, following the standard analysis procedure1 and using the version 2.0 tuned background 2 . The spectrum was consistent with the typical cosmic X-ray background spectrum and showed no hint of signal from V1647 Ori.

\section{Image Analysis}

Figure 1 shows an XIS color image of the V1647 field, combining all the XIS detectors $(0+1+3)$. The XISs detected three bright peaks at the center, top, and bottom of the image and four faint sources at $\sim 1.5^{\prime}$ north-east, $4^{\prime}$ south-east, $4^{\prime}$ south, and $8^{\prime}$ south-west from the central source. The bright sources to the north and south and the faint source to the south-east each have only one corresponding X-ray source in the 2002 Chandra observation, respectively (source 14, source 8, and source 23 in Simon et al. 2004) within the Suzaku pointing uncertainty $\left(\lesssim 20^{\prime \prime}\right) \sqrt[3]{3}$. We measured their source positions from a $0.5-10 \mathrm{keV}$ XIS image smoothed with the Gaussian function with $\sigma=2$ image pixel $\left(16.7^{\prime \prime}\right)$ by weighting photon counts within a $3 \times 3$ pixel region centered at the peak pixel. These positions were offset, on average, by $(\Delta$ R.A., $\Delta$ Dec. $)=\left(-8.3^{\prime \prime},-2.2^{\prime \prime}\right)$ from the coordinates in Simon et al. (2004), with a standard deviation of $2.0^{\prime \prime}$. We shifted the Suzaku image frame to match the Chandra coordinates.

After these corrections, the absolute coordinates of the central bright source $\left(\alpha_{2000}, \delta_{2000}\right)$ $=\left(5^{h} 46^{m} 13.35^{s},-00^{d} 06^{\prime} 5.7^{\prime \prime}\right)$ correspond to those of V1647 Ori within the $3 \sigma$ error range. Faint Chandra sources around V1647 Ori $(9,13,15)$ are on the outskirts of the PSF and do not show any apparent local peaks at their locations in the Suzaku image. In the Chandra

\footnotetext{
${ }^{1}$ http://heasarc.gsfc.nasa.gov/docs/suzaku/analysis/abc/

${ }^{2} \mathrm{ftp}$ // /legacy.gsfc.nasa.gov/suzaku/data/background/pinnxb_ver2.0_tuned/

${ }^{3}$ suzakumemo-2007-04 - http://www.astro.isas.ac.jp/suzaku/doc/
} 
observation in September 2008 (ObsID: 9915), these sources were far weaker than (had $\lesssim 3 \%$ of the photon counts of) V1647 Ori. Their contamination of V1647 Ori in the Suzaku data would therefore appear to be minimal. The soft source to the north-east from V1647 Ori is probably a blend of sources 20 and 21 in Simon et al. (2004), while the very hard source to the south is a blend of sources 7, 10, and 11, which are members of SSV 63, a cluster of class I protostars.

\section{Timing and Spectral Analysis}

The north-east source heavily contaminated the V1647 Ori region below $\sim 1 \mathrm{keV}$, but its contamination was negligible above $4 \mathrm{keV}$. To minimize the contamination and maximize the photon statistics, we defined a source region with a $1.5^{\prime}$ radius circle, excluding the circular region within $40^{\prime \prime}$ of the north-east source (see the right panel of Figure11). To cancel out the remaining contamination, we defined a background region that is symmetric to the source region with respect to the north-east source. The background-subtracted light curves and spectra presented in Figure 2-4 were generated from the events within these regions.

Figure 2 shows the background subtracted X-ray light curve of V1647 Ori covering the energy range $1-8 \mathrm{keV}$, combining all the XIS $(0+1+3)$ data, and using a bin size of $2 \mathrm{ks}$. The few missing data points are time periods where few good-time intervals $(<1 \%)$ were available. The count rate varied strongly, by a factor of $\gtrsim 18$, during the observation (count rate of the maximum bin: $\sim 0.11$ counts $\mathrm{s}^{-1}, 1 \sigma$ upper limit count rate of the minimum bin: $\sim 6 \times 10^{-3}$ counts $\left.\mathrm{s}^{-1}\right)$. The $\mathrm{X}$-ray light curve shows a gradual decrease, to a level consistent with zero flux, over the first $\sim 60 \mathrm{ks}$ of the observation, then a sharp increase in flux at $\sim 60$ ksec followed by a decay with significant spikes and dips.

We produced background subtracted soft band (1-4 keV) and hard band (4-8 keV) light curves and calculated the hardness ratio (HR) defined by $(H-S) /(H+S)$, where $S$ and $H$ are the soft and hard band count rates, respectively (bottom panel of Figure 2). The $\mathrm{HR}$ was $\sim-0.5$ between $10 \mathrm{ksec}$ and $60 \mathrm{ksec}$ into the observation and increased to $\sim 0.0$ after $\sim 60 \mathrm{ksec}$, remaining at that level for the rest of the observation duration. The abrupt flux increase at $\sim 60 \mathrm{ksec}$, accompanying an HR increase, may be explained by an increase in $k T$ (see also spectral analysis in the latter paragraphs), while fluctuations in flux after that, which are apparently uncorrelated with the HR, caused by changes in the plasma emission measure. Such flux and HR variations were seen in the XMM-Newton observation in 2004, as well (Grosso et al. 2005).

We merged spectra of the FI sensors and produced time-averaged FI and BI spectra 
(Figure 3). All the spectra in our analysis were binned so as to obtain at least 20 photon counts per bin. We ignored energy bins below $1.4 \mathrm{keV}$, where contamination from the northeast source exceeds the net signal from V1647 Ori. The resulting spectra reveal a strong fluorescent iron line at $6.4 \mathrm{keV}$ from quasi-neutral iron atoms, as well as the helium-like iron line at $6.7 \mathrm{keV}$, which is a signature of hot plasma. These features can be seen clearly in the Fe K $\alpha$ line region of the merged XIS spectra (right panel of Figure 3). The spike at $5.9 \mathrm{keV}$ possibly originates from contamination of the ${ }^{55} \mathrm{Fe}$ calibration source scattering over the CCD chips.

The spectrum is well fit by a model consisting of a single-component, optically thinthermal plasma emission (APEC) combined with a narrow Gaussian line with the center energy fixed at $6.4 \mathrm{keV}$ for the iron fluorescent emission, suffering photoelectric absorption by neutral gas (wabs). The best-fit parameters are listed in the model "i" in Table 1 . The best-fit plasma temperature $(\sim 4.1 \mathrm{keV})$, elemental abundance $(\sim 0.50$ solar $)$, hydrogen column density $\left(\sim 3.6 \times 10^{22} \mathrm{~cm}^{-2}\right)$, and the average observed flux $\left(\sim 4.4 \times 10^{-13} \mathrm{ergs} \mathrm{cm}^{-2} \mathrm{~s}^{-1}\right.$ between $0.5-8 \mathrm{keV}$ ) are similar to those inferred for V1647 Ori during the XMM-Newton observation in 2004 (Grosso et al. 2005). In the 2.8-8.0 keV band, the absorption corrected X-ray luminosity during the Suzaku observation $\left(\log L_{\mathrm{X}} \sim 30.9 \mathrm{ergs} \mathrm{s}^{-1}\right.$ ) was twice that seen during the XMM-Newton observation $\left(\log L_{\mathrm{X}} \sim 30.5 \mathrm{ergs} \mathrm{s}^{-1}\right)$. One remarkable difference, however, is the firm detection of the iron fluorescent line at $6.4 \mathrm{keV}$. The equivalent width (EW) in 2008, $\sim 600 \mathrm{eV}$, was about a factor of 6 higher than that found from the marginal detection of the iron fluorescent line during the XMM-Newton observation in 2004.

Because the spectrum changed so dramatically near the midpoint of the Suzaku exposure, we divided the observation into two phases, before and after $56 \mathrm{ksec}$ from the observation start, as shown at the top of Figure 2. We simultaneously fit these spectra with their elemental abundances tied. The best-fit results are shown in the model "ii" in Table 1 and Figure 4. The plasma temperature was significantly lower in the early phase, while the other parameters, especially the hydrogen column density, do not appear to vary significantly. This would suggest that the plasma heated up and/or that a very hot plasma emerged at $\sim 56 \mathrm{ksec}$ after the start of the observation. The best-fit EW of the iron fluorescent line was huge, close to $1.5 \mathrm{keV}$, during the "early" phase though the error was large enough to include the best-fit EW in the "late" phase. The model "iii" in Table 1 with their absorption and abundance parameters tied gives a similar result. Such large fluorescent iron line EWs have been observed from spatially extended X-ray reflection sources that do not contaminate emission from their irradiating sources (e.g., Koyama et al. 1996; Corcoran et al. 2004). 


\section{Discussion}

The Suzaku observation was performed between $\sim 40$ and $\sim 280$ days after the onset of the new accretion outburst from V1647 Ori. The first (2004) XMM-Newton observation of V1647 Ori during its 2003-2005 outburst was performed with similar timing, 4 months after outburst onset, with net exposure time (37-39 ksec) similar to that of the Suzaku observation. Since these two observatories have similar effective areas in the $0.5-10 \mathrm{keV}$ energy range, these observations are well suited to comparing the X-ray properties of V1647 Ori between the 2003 and 2008 outbursts.

The flux of X-ray emission from V1647 Ori strongly varied, by at least a factor of 18, during the Suzaku observation. The flux bottom is not well constrained given the contamination from the north-east soft source, but the XMM-Newton observation in 2004 showed a similar range of variation of between $0.5-12 \times 10^{-13} \mathrm{ergs}^{-2} \mathrm{~s}^{-1}$. Both observations showed similar abrupt flux increases with correspondingly HR increases and no significant HR variations after the increases. The plasma parameters derived from the Suzaku spectra were similar to the best-fit result of the XMM-Newton spectrum (model 1 in Table 1 . Grosso et al. 2005). These similarities suggest that the new outburst in 2008 was driven by a mechanism similar to that of the outburst which started in 2003 October and lasted for $\sim 2$ years. Similarities between the 2003-2005 and latest outbursts are also seen at near-infrared wavelengths (Aspin et al. 2009).

While V1647 Ori displayed strikingly similar X-ray activity patterns during its two mass accretion outbursts, there appears to be a very wide range of X-ray behavior and pre/postoutburst X-ray luminosities among the other FUor and EXor systems observed in X-rays at the onset of and/or during eruptions. The FUor systems FU Ori and V1735 Ori emit strong X-rays at levels of $\log L_{\mathrm{X}} \sim 31 \mathrm{ergs} \mathrm{s}^{-1}$ from hot plasma, $k T>5 \mathrm{keV}$ (Skinner et al. 2006, 2009), similar to that seen from V1647 Ori. On the other hand, several FUor and EXor systems did not show remarkable X-ray activity associated with their mass accretion outbursts (EXor - V1118 Ori: Audard et al. 2009, FUor - V1057 Cyg and V1515 Cyg: Skinner et al. 2009). These particular X-ray characteristics are apparently not directly related to the optical outburst criteria that are used to classify objects as FUors or EXors (e.g., outburst duration). The X-ray behavior during outburst therefore might be more reasonably connected to the other YSO characteristics, such as rotational velocity and/or strength of the magnetic field.

The Suzaku spectra in 2008 showed an impressively strong fluorescent iron K $\alpha$ line feature at $6.4 \mathrm{keV}$. Such a large EW value has been reported only from a few young stellar objects $\left(\mathrm{X}_{E}\right.$ or IRS 7B: Hamaguchi et al. 2005, IRS1: Skinner et al. 2007, V1486 Ori: Czesla \& Schmitt 2007). The observed iron fluorescent EWs are more than 10 times as 
large as that for a $4 \mathrm{keV}$ plasma irradiating an infinite plane of solar iron abundance in a Monte-Carlo simulation ( $\lesssim 60 \mathrm{eV}$, Drake et al. 2008), so that special physical conditions would be required to explain such a large EW. Plausible conditions that can elevate the EWs are (1): supersolar iron abundance, (2): re-emission by a neutral absorber, e.g., a flaring disk and/or infalling envelope, (3): time-delay effect of fluorescence intensified by radiation from a magnetic flare, (4): line-of-sight obscuration of the irradiating plasma, (5): excitation by non-thermal electrons produced by a magnetic flare (e.g., Osten et al. 2007; Czesla \& Schmitt 2007). Among them, the conditions (3) and (5) would require a big flare to produce the large EW, and which was not observed during the Suzaku observation. The condition (2) can only increase the EW by a factor of 2 . The condition (1) is unlikely because, in this condition, the photosphere and/or disk abundances would have had to increase by a factor of six between 2004 and 2008. We thus favor the condition (4) as the most likely explanation.

Assuming the presence of a reflector with solar iron abundance on an infinite plane, $\sim 90 \%$ of the plasma has to be blocked from our direct view by an optically thick absorber in order to explain the large iron fluorescent line EW measured for V1647 Ori in 2008 by Suzaku. Such a geometry is plausible if most of the plasma is hidden behind the stellar core and/or disk (see the discussion in Hamaguchi et al. 2005). We note that Aspin et al. (2009) demonstrated a change in the $r^{\prime}$ band morphology of the reflection nebula associated with V1647 Ori (McNeil's Nebula) between 2004 and 2008, and suggested that dust obscuration close to V1647 Ori may play an important role in defining the observed morphology of the nebula. The circumstellar gas structure very close to the stellar core may have changed between 2004 and 2008, and this change may have altered both the reflection paths of X-ray emission and the openings in visible light between the star and McNeil's nebula.

We greatly appreciate the Suzaku review committees positive response to our DDT request. This work is performed while K.H. was supported by the NASA's Astrobiology Institute (RTOP 344-53-51) to the Goddard Center for Astrobiology. J.K.'s research on X-rays from erupting YSOs is supported by NASA/GSFC XMM-Newton Guest Observer grant NNX09AC11G to RIT.

Facilities: Suzaku (XIS)

\section{REFERENCES}

Anthony-Twarog, B. J. 1982, AJ, 87, 1213 
Aspin, C. 2008, IAU Circ., 8969, 2

Aspin, C., \& Reipurth, B. 2009, AJ, 138, 1137

Aspin, C. et al. 2009, ApJ, 692, L67

Audard, M., et al. 2009, A\&A in print (arXiv:0912.3224)

Audard, M., Güdel, M., Skinner, S. L., Briggs, K. R., Walter, F. M., Stringfellow, G., Hamilton, R. T., \& Guinan, E. F. 2005, ApJ, 635, L81

Corcoran, M. F. et al. 2004, ApJ, 613, 381

Czesla, S., \& Schmitt, J. H. H. M. 2007, A\&A, 470, L13

Drake, J. J., Ercolano, B., \& Swartz, D. A. 2008, ApJ, 678, 385

Grosso, N. 2006, in ESA Special Publication, Vol. 604, The X-ray Universe 2005, ed. A. Wilson, p. $51-56$

Grosso, N., Kastner, J. H., Ozawa, H., Richmond, M., Simon, T., Weintraub, D. A., Hamaguchi, K., \& Frank, A. 2005, A\&A, 438, 159

Hamaguchi, K., Corcoran, M. F., Petre, R., White, N. E., Stelzer, B., Nedachi, K., Kobayashi, N., \& Tokunaga, A. T. 2005, ApJ, 623, 291

Hartmann, L., \& Kenyon, S. J. 1996, ARA\&A, 34, 207

Itagaki, K., Nakano, S., \& Yamaoka, H. 2008, IAU Circ., 8968, 2

Kastner, J. H. et al. 2004, Nature, 430, 429

Kastner, J. H. et al. 2006, ApJ, 648, L43

Kokubun, M. et al. 2007, PASJ, 59, 53

Koyama, K., Maeda, Y., Sonobe, T., Takeshima, T., Tanaka, Y., \& Yamauchi, S. 1996, PASJ, 48, 249

Koyama, K. et al. 2007, PASJ, 59, 23

Mitsuda, K. et al. 2007, PASJ, 59, 1

Ojha, D. K., Ghosh, S. K., Kaurav, S. S., Bhatt, B. C., Sahu, D. K., \& Tej, A. 2008, IAU Circ., 9006, 1 
Osten, R. A., Drake, S., Tueller, J., Cummings, J., Perri, M., Moretti, A., \& Covino, S. 2007, ApJ, 654, 1052

Preibisch, T. et al. 2005, ApJS, 160, 401

Serlemitsos, P. J. et al. 2007, PASJ, 59, 9

Simon, T., Andrews, S. M., Rayner, J. T., \& Drake, S. A. 2004, ApJ, 611, 940

Skinner, S. L., Simmons, A. E., Audard, M., Güdel, M. 2007, ApJ, 658, 1144

Skinner, S. L., Briggs, K. R., \& Güdel, M. 2006, ApJ, 643, 995

Skinner, S. L., Sokal, K. R., Güdel, M., \& Briggs, K. R. 2009, ApJ, 696, 766

Takahashi, T. et al. 2007, PASJ, 59, 35

Telleschi, A., Güdel, M., Briggs, K. R., Audard, M., \& Palla, F. 2007, A\&A, 468, 425 
Table 1. Spectral Fits

\begin{tabular}{|c|c|c|c|c|c|c|c|c|}
\hline Model & Phase & $\begin{array}{c}k T \\
(\mathrm{keV})\end{array}$ & $\begin{array}{c}Z \\
\text { (solar) }\end{array}$ & $\begin{array}{c}N_{\mathrm{H}} \\
\left(10^{22} \mathrm{~cm}^{-2}\right)\end{array}$ & $\begin{array}{c}\text { Line Flux }{ }^{a} \\
\left(10^{-6} \mathrm{ph} \mathrm{cm}^{-2} \mathrm{~s}^{-1}\right)\end{array}$ & $\begin{array}{l}\text { Line } \mathrm{EW}^{b} \\
\quad(\mathrm{eV})\end{array}$ & $\begin{array}{c}\log L_{\mathrm{X}}^{c} \\
\left(\operatorname{ergs~s}^{-1}\right)\end{array}$ & $\chi^{2}$ (d.o.f.) \\
\hline $\mathrm{i}$ & whole & $4.1(3.2-5.6)$ & $0.50(0.31-0.73)$ & $3.6(2.9-4.4)$ & $2.7(1.6-3.8)$ & 588 & $31.1 / 30.9 / 31.3$ & $1.03(85)$ \\
\hline \multirow[t]{2}{*}{ ii } & early & $1.9(1.4-2.7)$ & $0.65(0.36-1.02)$ & $5.1(3.7-7.1)$ & $2.1(0.66-3.6)$ & 1328 & $31.3 / 30.7 / 31.4$ & $1.00(84)$ \\
\hline & late & $5.3(3.8-7.5)$ & $=$ early & $3.6(2.9-4.5)$ & $4.5(2.3-6.8)$ & 549 & $31.2 / 31.2 / 31.5$ & $\ldots$ \\
\hline \multirow[t]{2}{*}{ iii } & early & $2.3(1.8-3.2)$ & $0.62(0.32-0.97)$ & $4.0(3.3-4.9)$ & $2.4(0.33-3.5)$ & 1355 & $31.1 / 30.7 / 31.2$ & $1.02(85)$ \\
\hline & late & $4.7(3.5-6.8)$ & $=$ early & $=$ early & $3.8(2.1-7.0)$ & 459 & $31.2 / 31.2 / 31.5$ & $\ldots$ \\
\hline $1^{d}$ & 2004 April 4 & $3.0(2.4-3.9)$ & $0.8(0.5-1.3)$ & $2.9(2.5-3.4)$ & $\ldots$ & 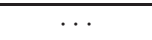 & $30.8 / 30.5 / 31.0$ & $1.11(129)$ \\
\hline
\end{tabular}

Note. - ${ }^{a}$ Total photon flux in the narrow Gaussian line with a fixed center at $6.4 \mathrm{keV} .{ }^{b}$ Measured with the xspec12 command "eqw range 0 Gaussian_component_ID". ' Absorption corrected luminosity between $0.5-2.8 / 2.8-8 / 0.5-8 \mathrm{keV}$, assuming $d=400$ pc. ${ }^{d}$ Model \#1 in Table 1 of Grosso et al. (2005). The parentheses between the second and fifth columns show $90 \%$ confidence ranges. 

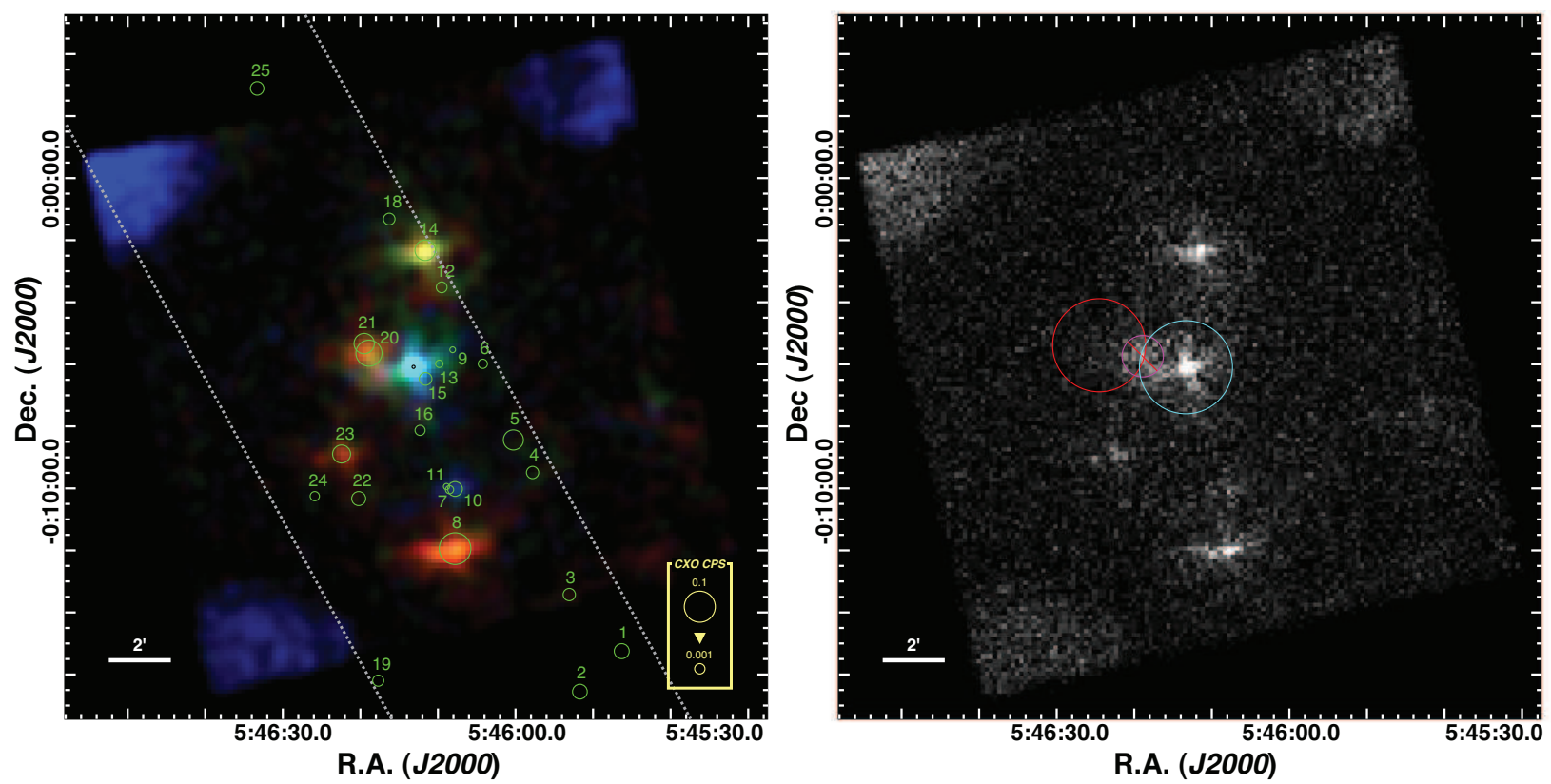

Fig. 1.- Left: XIS 0+1+3 color image of the V1647 Ori field (red: $0.5-2 \mathrm{keV}$, green: 2-4 keV, blue: 4-10 keV) after correcting the absolute coordinates using a Chandra result (Simon et al. 2004). The image was smoothed by the Gaussian function with $\sigma=3$ image pixels $\left(\sim 25^{\prime \prime}\right)$ and displayed in a linear scale for the 5 counts pixel ${ }^{-1}$ range in each color, offset at the background level determined from a source free region. The extended blue areas at the 3 detector corners are ${ }^{55} \mathrm{Fe}$ calibration sources. Green circles with numbers and a black circle at the center (V1647 Ori) are X-ray source positions detected in the Chandra observation on 2002 November 14 (Simon et al. 2004; Kastner et al. 2004). Radii of these circles are proportional to Chandra photon count rates between $0.5-10 \mathrm{keV}$ in logarithmic scale, as shown at the bottom right corner. The grey dots show the Chandra ACIS-S FOV. Right: monochromatic XIS $0+1+3$ unsmoothed image of the same field between $0.5-10 \mathrm{keV}$. The cyan and red circles show source and background regions, respectively; the region within the magenta circle was excluded from these source and background regions. 


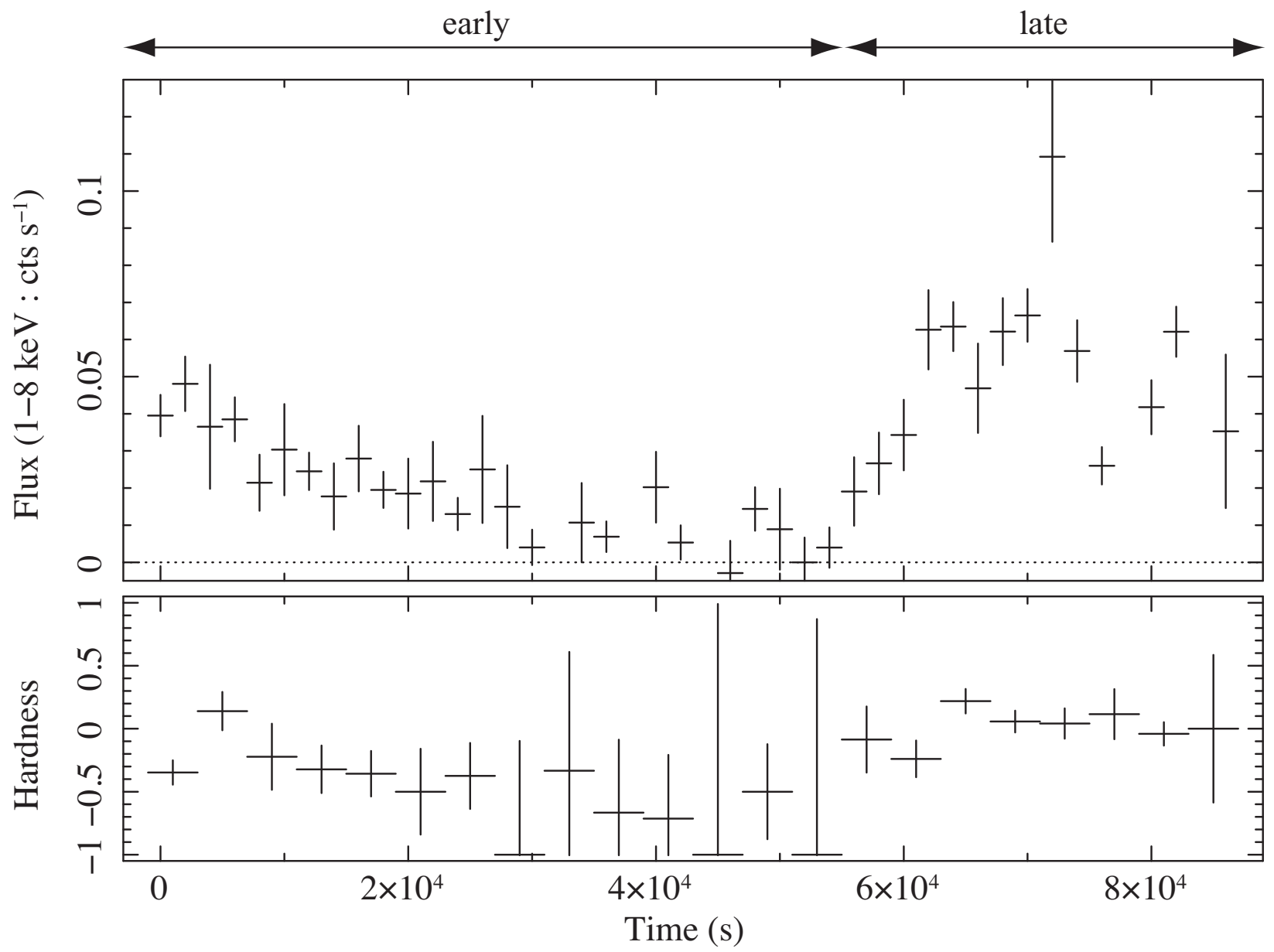

Fig. 2.- Top panel: Background subtracted light curve of V1647 Ori between 1-8 keV, produced from the XIS $0+1+3$ data. Each bin has $2 \mathrm{ksec}$. Bottom panel: The hardness ratio curve with $4 \mathrm{ksec}$ bins defined by $(H-S) /(H+S)$, where $H$ and $S$ are the hard (4-8 keV) and soft $(1-4 \mathrm{keV})$ band count rates, respectively. The label above the top panel shows intervals defined for phase-resolved spectral analysis. The origin of the x-axis is 2008 October $8,14^{h} 33^{m} 10^{s}$ in UT. 

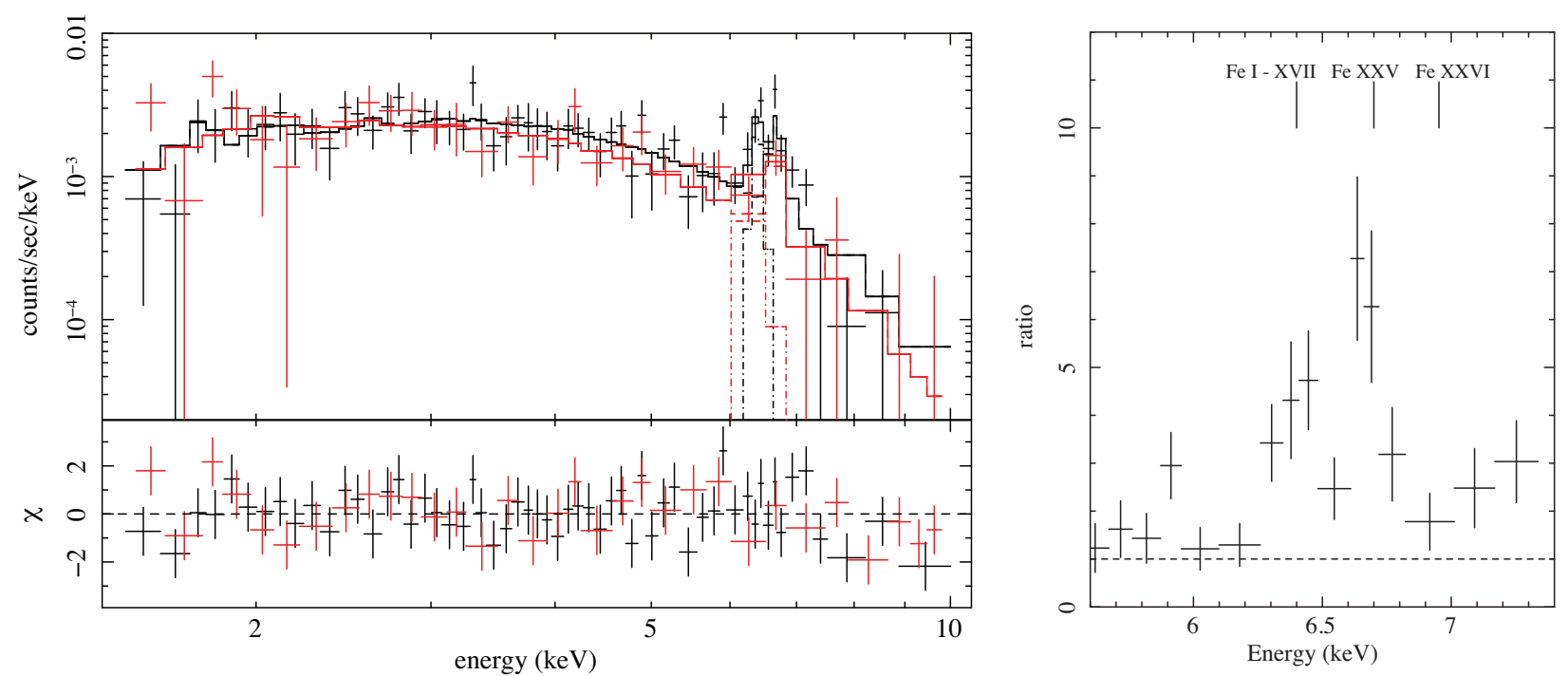

Fig. 3.- Left Top: XIS FI (black) and BI (red) spectra of V1647 Ori. The solid lines show the best-fit result of the spectra by a model of 1-temperature thin-thermal plasma emission (APEC) with a Gaussian function at $6.4 \mathrm{keV}$ for the iron fluorescent line, suffering photoelectric absorption by quasi-neutral gas. Left Bottom: residuals of the $\chi^{2}$ values from the best-fit model. Right: XIS $0+1+3$ spectrum near the iron $K$ line region. The vertical axis shows the ratio of the emission against the continuum in the best-fit model (model "i" in Table 1). Important emission lines are marked by lines at their rest energies.
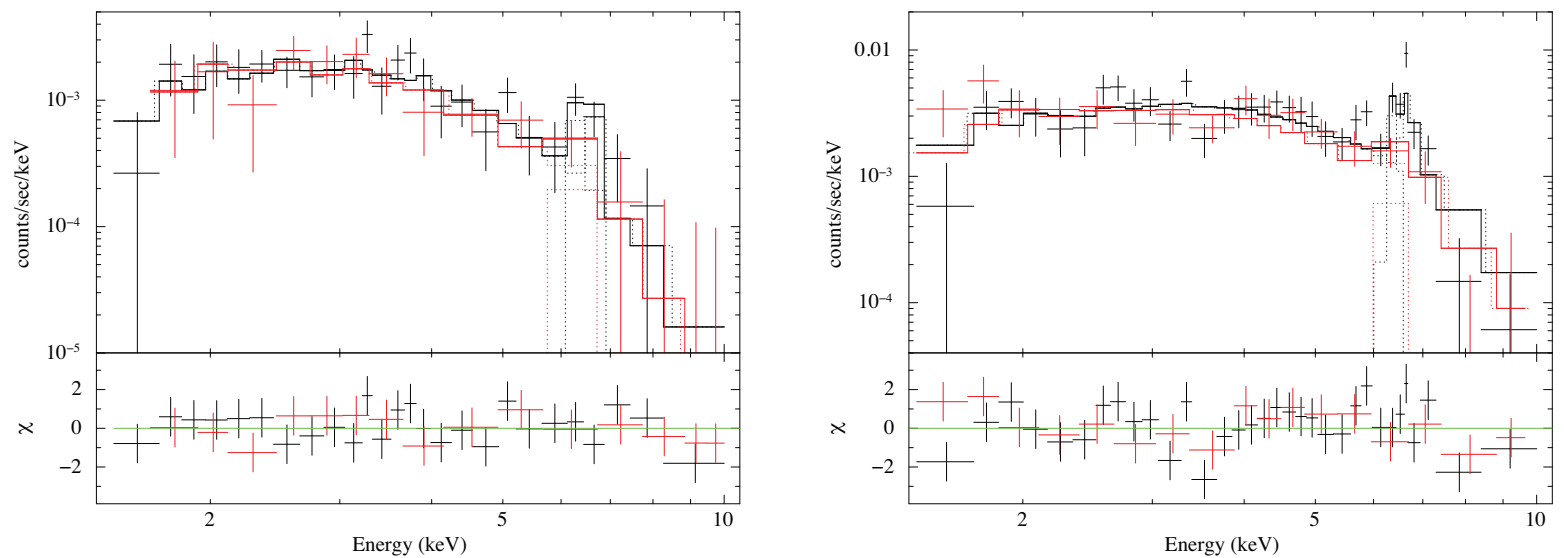

Fig. 4.- X-ray spectra of the early (left) and late (right) phases (model "ii"). 